\title{
XIII. Beiträge zur Kenntniss der chemischen Constitution und der Genese der natürlichen Eisensulfate. I.
}

\author{
Von \\ Rudolf Scharizer in Czernowitz. \\ (Mit $\uparrow$ Textfigur.)
}

\begin{abstract}
Seit mehr als sieben Jahren beschätige ich mich mit der Frage nach der Genese der natürlichen Eisensulfate. Ich versuchte, diese Frage mittelst synthetischer Versuche zu lösen, bei deren Ausführung ich mein Hauptaugenmerk bes̀onders darauf richtele, die Versuchsbedingnisse möglichst genau den in der Natur obwaltenden Verhältnissen anzupassen. Es wurde vor allem die Anwendung von höheren Temperaturen vermieden, weil ja dieselben bald fördernd, bald hindernd in den Gang der chemischen Reactionen eingreifen und die Resultate, welche ich zu erlangen strebte, nicht von vornherein ihre Anwendbarkeit auf die Erklärung der Vorgänge in der Natur infolge der Versuchsbedingnisse verlieren sollten. Da nur dié Zeit den Einfluss der Wärme theilweise zu ersetzen vermag, so dehnlen sich viele meiner Versuche uber Monate und Jahre aus. Dies, sowie der Umstand, dass wahrend der Arbeit stetig neue Probleme auftauchten, deren Lösung doch wenigstens versucht werden sollte, verhinderten eine fruhere Publication der Untersuchungsergebnisse, von denen einige bis in das Jahr 1890 zuruckreichen, und veranlasst mich jetzt, anstatt mit einer abgeschlossenen grossen Arbeit mit einer Reihe von kleineren Mittheilungen vor die Oeffentlichkeit zu treten. Diese erste Mitlbeilung ist dew Eisenvitriol und allen jenen Erscheinungen, dieseine Oxydalionbegleiten, gewidmet.
\end{abstract}

\section{Der Eisenvitriol.}

$\$ 1$. Der Wassergehalt des Eisenvitriols. Trotzdem der Eisenvitriol schon vielen Forschern als Untersuchungsmaterial gedient hat, so sind doch die Bedingungen, unter welchen er sein Wasser verliert, noch 
immer nicht vollkommen klargelegt. Allgemein verbreitet in der Literatur ist die Angabe, dass der Eisenvitriol 6 Moleküle $=38,88 \%$ bei ca. $80^{\circ} \mathrm{C}^{1}$ ) verliere, und dass das letzte Molekul, dem Liebig ${ }^{2}$ ) den Namen Hal hy dratwasser gegeben hat, im Vacuum nach $G m e l i n-K r a u t^{3}$ ) schon bei $140^{\circ}$, nach Mitscherlich 4) zwischen $200^{\circ}$ und $300^{\circ}$, nach Graham ${ }^{5}$ ) bei $280^{\circ}$ aus der Verbindung austrete.

Von diesen Angaben weichen die Ergebnisse der von mir allerdings unter anderen Versuchsbedingungen angestellten Versuche in einigen Punkten ab. Der Eisenvitriol wurde nämlich nicht im Vacuum, sondern in einem kupfernen Trockenkasten erhilzt. Die so beobachteten Zahlenwerthe geben daher nicht den der betreffenden Temperatur entsprechenden Wasserverlust direct an, weil ja neben der Wasserabgabe auch die Oxydation des Eisenvitriols einhergeht. Dieselbe beginnt erst bei einem Erhitzen auf $60^{\circ}$ merkbar zu werden. Man kann aber diesen störenden Einfluss der Oxydation dadurch leicht eliminiren, dass man der Probe von Zeit zu Zeit kleine Mengen zur Bestimmung des jeweiligen Eisenoxydulgebaltes entnimmt. Den Gang dieser allmählich fortschreitenden Oxydation soll der Versuch III in nachstehender Tabelle demonstriren. Die Versuche I und II sind der Controle wegen angestellt worden.

Tabelle I.

\begin{tabular}{|c|c|c|c|c|c|c|c|c|c|}
\hline \multirow[b]{2}{*}{$t$} & \multicolumn{2}{|c|}{ I. } & \multicolumn{2}{|c|}{ II. } & \multicolumn{4}{|c|}{111.} & \\
\hline & $\begin{array}{l}\text { Gew.- } \\
\text { Verl. }\end{array}$ & $\left|\begin{array}{c}\mathrm{FeO}- \\
\text { Gehalt }\end{array}\right|$ & $\begin{array}{l}\text { Gew.- } \\
\text { Verl. }\end{array}$ & $\mid \begin{array}{r}\mathrm{FeO}- \\
\text { Gehalt }\end{array}$ & $\begin{array}{l}\text { Gew.- } \\
\text { Verl. }\end{array}$ & $\mid \begin{array}{c}\mathrm{FeO}- \\
\text { Gehalt }\end{array}$ & \begin{tabular}{|c} 
Aufge- \\
nomma. \\
0
\end{tabular} & $\mathrm{H}_{2} \mathrm{O}$ & \\
\hline 400 & 19,62 & - & 19,52 & - & 19,58 & 25,90 & 0 & 19,58 & $3 \mathrm{Mol} .=19,410 \%$ \\
\hline 60 & 17,75 & - & 17,98 & - & 17,89 & 23,15 & 0,30 & 18,19 & \\
\hline 80 & 0,38 & - & 0,37 & - & 0,35 & 15,13 & 0,90 & 1,25 & $3 \mathrm{Mol} .=19,41$ \\
\hline 100 & 0,36 & 1,37 & 0,87 & 4,71 & 0,72 & 3,86 & 1,21 & 1,93 & $\frac{2}{6} \mathrm{Mol} .=2,16$ \\
\hline- & 38,11 & - & 38,74 & - & 38,54 & - & 2,41 & 40,95 & \\
\hline O-Zunahme & 2,74 & - & 2,35 & - & 2,41 & - & - & - & \\
\hline- & 40,85 & - & 41,09 & - & 40,95 & - & - & - & \\
\hline
\end{tabular}

Die Oxydation des Eisenvitriols ist also bei $100^{\circ}$ nahezu vollendet. Das Pulver hat nun eine licht gelbbraune Farbe angenommen, deren Intensităt sicb beim stärkeren Erhitzen nicht mehr wesentlich änderte. Gleich-

1) In Graham-Otto's Lehrbuch der Chemie, 4. Aufl., IV. Ablh. 1, 590 ist als Temperatur, bei welcher diese sechs Moleküle weggehen, $115^{0}$ angegeben.

2) Ann. f. Pharm. 26, 144.

3) Handbuch d. anorg. Chemie s, 339.

4) Pogg. Ann. 18, 152.

5) Phil. Mag. J. 3, 421. 
Beitr. zur Kenntn. der chem. Constit. u. der Genese der nalürl. Eisensulfate. I. 211

zeitig trelen bis 1000 auch circa 6\% $6_{6}^{2}$ Moleküle Wasser, d. i. 41,00\% $\mathrm{H}_{2} \mathrm{O}$ aus der Verbindung aus. $\frac{1}{6}$ Molekül $=1,08 \%$ entweicht dann allmählich bis $300^{\circ}$; die restlichen $\frac{3}{6}$ Moleküle $=3,24 \%$ werden dagegen erst durch Gluhen ausgetrieben.

Nachstehende Tabelle II fasst die Ergebnisse eines Versuches zusammen, durch welchen das allmähliche Entweichen des Halhydratwassers festgestellt wurde. Da die Substanz jeder der angegebenen Temperaturen so lange ausgesetzt blieb, bis Gewichtsconstanz eingetreten war, so dehnle sich diese Versuchsleihe über vier Monate aus.

Tabelle II.

\begin{tabular}{|c|c|c|c|c|c|c|c|c|c|c|}
\hline$t$ & $40^{0}$ & $60^{0}$ & $80^{0}$ & $100^{\circ}$ & $120^{\circ}$ & $140^{0}$ & $160^{\circ}$ & $180^{\circ}$ & $200^{\circ}$ & $220^{\circ}$ \\
\hline Verlust & $19,64 \%$ & $16,500 / 0$ & $1,29 \%$ & $0,420 / 0 \mid$ & $0,510 \%$ & $0,19 \%$ & $0,06 \%$ & $0,24 \%$ & $0,29 \%$ & 0,190 \\
\hline & $t$ & 2400 & 260 & & $280^{\circ}$ & 3000 & \multicolumn{2}{|c|}{$360^{\circ}$} & Glühen & \\
\hline \multicolumn{2}{|c|}{ Verlust } & $0,22 \%$ & $0,03^{\circ}$ & & $0,09 \%$ & $0,12 \%$ & \multicolumn{2}{|c|}{$0,06 \%$} & $3,21 \%$ & \\
\hline
\end{tabular}

Der Glühverlust über $360^{\circ}$ wurde hier durch Glühen mit Bleioxyd bestimmt. Bei einem weileren Versuche wurde ein Theil der bei $360^{\circ}$ getrockneten Substanz zur Bestimmung des Eisenoxydulgebaltes verwendet, - allerdings mit negativem Erfolge -, ein anderer Theil wurde wieder mit Bleioxyd geglutht, und an einem drillen Theile bestimmte Herr Glucksman n, seiner Zeit Assistent am hiesigen chemischen Universitätslaboratorium, den Wassergehalt durch Glühen in einer mit Bleichromat gefullten Verbrennungsröhre und Auffangen des entweichenden Wassers im Chlorcalciumrohre.

Im ersten Falle betrug der Gewichtsverlust auf die ungetrocknete Substanz bezogen $3,30 \%$, im anderen Falle die Menge des aufgefangenen Wassers auf die ungetrocknete Substanz bezogen $3,33 \%$.

Dieselbe Menge Wasser, welche der Eisenvitriol bei $40^{\circ}$ abgiebt, verliert derselbe auch in wenigen Tagen über Schwefelsäure. Die Schnelligkeit der Wasserabgabe hängt naturlich vor allem von der Menge des verwendeten Eisenvitriols ab, dann aber auch von dem Umstande, ob derselbe in Stucken oder in Pulverform der wasserentziehenden Einwirkung der Schwefelsäure uberantwoṛtet wurde. Wird der Eisenvitriol hierauf noch weiter im Exsiccator belassen, so vergrössert sich der Gewichtsverlust noch um einige Procente.

So verloren z. B. 1,2335 g Eisenvitriol in kleinen Stucken uber Schwefelsäure 
am 1. Tage, d. i. in 24 Stunden $0,0538 \mathrm{~g}$

$\begin{aligned}-2 .- & 0,0613- \\ -3 .- & 0,0619- \\ -4 .- & 0,0579- \\ -5 .- & 0,0068- \\ -6 .- & 0,0015- \\ -7 .- & 0,0010-\end{aligned} \quad \Sigma=0,2417 \mathrm{~g}=3$ Mol. $[0,2394 \mathrm{~g}]$

In den darauffolgenden 13 Wochen verlor der Eisenvilriol durchschnittlich $0,0031 \mathrm{~g}$ per Woche und im Zeitraume vom 1. März 1897 bis 26. Januar 1898 nabm das Gewicht uberhaupt nur mehr um $0,0080 \mathrm{~g}$ ab. Mit diesem Tage wurde die Versuchsreihe abgebrochen. Der Gesammtgewichtsverlust des Eisenvitriols im Exsiccator betrug $0,3081 \mathrm{~g}=25,04 \%$, d. i. nahezu 4 Molekule; das wären $0,3192 \mathrm{~g}$ oder $25,88 \%$.

Da nach diesen Versuchen das Halhydratwasser zum Theile unter $360^{\circ}$, zum Theile uher $360^{\circ}$ entweicht, so könnte man daraus den Schluss ziehen, dass der Wasserstoff des Halhydratwassers zwei ungleich stark gebundenen Hydroxylmolekülen angehört, und darin auch einen Beweis für die Erlenmayer'sche Formel des Eisenvitriols $\underset{\mathrm{HO}-\mathrm{Fe}}{\mathrm{H}}>\mathrm{SO}_{4}+6$ aq sehen. Es ist aber zu bedenken, dass bei meinen Versuchen sich der Eisenvitriol auch oxydirte. Die Constitution des Pulvers entspricht schon bei $100^{\circ}$ nicht mehr vollkommen der Formel $\mathrm{FeSO}_{4}$ und bei höheren Temperaturen, sicherlich bei $360^{\circ}$, kommt der ganzen Masse, sobald man vom Wassergehalte absiehl, die Formel $\mathrm{Fe}_{2} \mathrm{~S}_{2} \mathrm{O}_{9}$ zu. Es ist mehr als wahrscheinlich, dass mit dieser durch die Oxydation bedingten Aenderung in der chemischen Zusammensetzung auch ein Wechsel in der Bindung des Wassers eintritt, so dass die Ergebnisse des Erhitzens uber $100^{\circ}$ nicht mehr auf das Ferrosulfat, sondern schon auf das Ferrisulfat zu beziehen sind.

Ich werde später Gelegenheit finden, nochmals auf diese Frage zurłuckzukommen.

Fur die nachfolgenden Untersuchungen ist wichtig festzuhalten, dass der Eisen vitriol 3 Moleküle $\mathrm{H}_{2} \mathrm{O}=19,44 \%$ rasch über Schwefelsäure verliert, dass 3 Molekule $\mathrm{H}_{2} \mathrm{O}=19,44 \%$ unter theilweiser Oxydation der Substanz zwischen $60^{\circ}$ und $80^{\circ}$ entweichen, dass $\frac{2}{6}$ Molekule Wasser circa zwischen $100^{\circ}$ und $160^{\circ}, \frac{1}{6}$ zwischen $160^{\circ}$ und $300^{\circ}$ weggehen, und dass vom oxydirten Eisenvitriol das letzte balbe Molekul erst durcb Gluhen abgespalten werden kann.

§ 2. Das Verstäuben des Eisenvitriols. Es ist allgemein bekannt, dass der Eisenvitriol beim Liegen an der Luft in ein weisses Pulver zerfällt, eine Erscheinung, welche man Verstäuben nennt.

Ueber die Endproducte dieser Verstäubung gehen jedoch die Meinungen der Forscher ziemlich weit auseinander. 
Nach Dammer ${ }^{1}$ ) verwilterl der Eisenvitriol an der Luft "unter oberflächlicher Oxydation zu basischem Ferrisulfat «.

In Graham-O (to's Lehrbuch der Chemie ${ }^{2}$ ) heisst es, dass der Eisenvitriol an trockener Luft in das wasserfreie Salz zerfalle.

Dana $\left.{ }^{3}\right)$ sagt: Exposed to the air becomes covered with a yellow powder, which is a ferric sulphat.

Endlich macht J. M. Mackin tosh am Schlusse seiner Arbeil: "Notes on some native Iron sulphates from Chili (') die Bemerkung, dass der Melanterit tbalsächlich leicht in $\mathrm{FeSO}_{4}+$ aq durch Wasserenlziehung unter Fernhaltung oxydirender Einflusse übergehe.

a) Um in diese Frage Klarheit zu bringen, analysirte ich einen kunstlichen Eisenvitriolkrystall, der uber funf Monate an freier Luft gelegen hatle und unter Beibehaltung seiner äusseren Gestalt sich ganz in eine weisse pulverige Substanz umgewandelt hatte. Die Zusammensetzung desselhen ist in nachstebender Tabelle unter a. angefuhrt.

$\begin{array}{lrrrc} & \text { 8. } & \text { Mol.-Quot.: } & \text { b. } & \text { Beob. - Rechn.: } \\ \mathrm{FeO} & 37,60 & 0,522 & 37,40 & +0,20 \\ \mathrm{SO}_{3} & 41,39 & 0,518 & 41,56 & -0,17 \\ \mathrm{H}_{2} \mathrm{O} & \frac{21,01}{100,00} & 1,167 & 21,04 & -0,03\end{array}$

Eisenoxyd konnte nicht nachgewiesen werden. Das Verhältniss von $\mathrm{SO}_{3}: \mathrm{H}_{2} \mathrm{O}$ ist hier anstatt $1: 7$ nur mehr $1: 2,25$. Es sind also unter der wasserentziehenden Einwirkung der Zimmerluft nicht nur jene 3 Molekule $\mathrm{H}_{2} \mathrm{O}$ aus der Verbindung ausgetreten, die der Eisenvitriol iber Schwefelsäure nach wenigen Tagen abzugeben pflegt, sondern die Wasserabgabe schritt noch weiter zur theilweisen Bildung der nächsten Stufe $\mathrm{FeSO}_{4}+$ aq vor. Die Zusammensetzung des analysirten, verstäublen Eisenvitriols lässt sich durch die Formel :

$$
5\left[\mathrm{FeSO}_{4}+4 \mathrm{aq}\right]+7\left[\mathrm{FeSO}_{4}+\mathrm{aq}\right]
$$

ausdrücken. Dieser Formel entspricht die sub b) angefuhrte Zusammensetzung.

Einen Prufstein für die Richtigkeit obiger Annahme lieferl auch das Verhalten des verstäubten Eisenvitriols beim allmählichen Erhitzen. Derselbe verlor:

$$
\begin{aligned}
\text { Uaber Schwefelsäure } & =0,05 \% \\
\text { bei } 80^{\circ} & =11,18 \\
-150 & =0,56 \\
-190 & =0,34
\end{aligned}
$$

1) Dammer, Handbuch der anorganischen Chemie 3, 328.

2) Lehrbuch der Chemie 5. Aufl. II, 4, 589.

3) System of Mineralogy, 6. Ed., 942.

4) Am. Journ. of Sc. III, 38, 245. Diese Zeitschr. 18, 671. 
Da die Oxydation des Eisenvitriols nach dem im vorhergehenden Paragraphen Gesagten bei $190^{\circ}$ zweifellos schon vollendet ist, so wären noch $4,18 \%$ als Aequivalent für die Gewichlszunahme des Pulvers in Folge der Oxydation dazuzurechnen. Der Gesammlverlust bis $190^{\circ}$ betruge dan $16,31 \%$ und der Verlust uber $190^{\circ}$ würde danu 4,70\% sein.

Gestutzt auf die im vorigen Abschnitte gemachten Erfahrungen sollen verlieren :

Tabelle III.

\begin{tabular}{|c|c|c|c|c|c|}
\hline & $30\left[\mathrm{FeSO}_{4}+4 \mathrm{aq}\right]$ & $42\left[\mathrm{FeSO}_{4}+\mathrm{aq}\right]$ & Summe & $\begin{array}{c}\text { Berechnet } \\
\mathrm{H}_{2} \mathrm{O} \%\end{array}$ & Beobacht. \\
\hline ¿ib.Schwefelsäure & $0 \mathrm{Mol}$. & $0 \mathrm{Mol}$. & 0 & - & - \\
\hline bei $80^{\circ}$ & $90-$ & $0-$ & 90 & $41,69)$ & \\
\hline-150 & $10-$ & $14-$ & 24 & $3,11\} 16,35$ & 16,31 \\
\hline bis 300 & $5-$ & 7 & 12 & $1,56)$ & \\
\hline \multirow[t]{2}{*}{ über 300} & $15-$ & $21-$ & 36 & 4,68 & 4,70 \\
\hline & 120 & 42 & 162 & 21,04 & 21,01 \\
\hline
\end{tabular}

Die Uebereinstimmung zwischen Beobachtung und Rechuung ist eine befriedigende.

b). Beim Zerreiben des verstäubten Krystalles beobachtete ich, dass derselbe aus einem härteren Kerne und einer weicheren Hülle bestand. Ich versuchte nun bei anderen verstäubten Krystallen Kern und Hulle durch gelindes Schutteln in einem feinmaschigen Messingsiebe zu trennen, was auch gelang.

Das durch das Messingsieb durchgefallene Pulver der weicheren Hulle halte folgende Zusammensetzung:

\begin{tabular}{lrrrc} 
& \multicolumn{1}{c}{ a. } & Mol.-Quol.: & \multicolumn{1}{c}{ b. } & Beob. - Rechn: \\
$\mathrm{FeO}$ & $4.0,81$ & 0,567 & 40,84 & $-0,03$ \\
$\mathrm{Fe}_{2} \mathrm{O}_{3}$ & 1,37 & 0,009 & 1,44 & $-0,07$ \\
$\mathrm{SO}_{3}$ & 46,49 & 0,581 & 46,46 & $-0,03$ \\
$\mathrm{H}_{2} \mathrm{O}$ & 11,33 & 0,630 & $\frac{11,36}{100,00}$ & $+0,07$
\end{tabular}

Das Verhältniss $\mathrm{H}_{2} \mathrm{O}: \mathrm{SO}_{3}=1,08: 1$. Der Verstäubungsprocess hat somit hier schon zur Bildung der fast reinen Verbindung $\mathrm{FeSO}_{4}+$ aq geführt. Ḍiesem Salze ist noch ein Ferrisulfat von einer Hohmannit-ähnlichen Zusammensetzung beigemischt. Denn der Schwefelsăureüberschuss und der beobachtete Eisenoxydgehalt stehen in einem molekularen Verhältnisse wie 1,55 : 1 [bei Hohmannit $=1,5: 1$ ]. Obwohl es dadurch keineswegs als bewiesen gelten soll, dass Hohmannit thalsächlich dem Pulver beigemengt isı ${ }^{1}$, , stelle ich dennoch den geringen. Eisenoxydgehalı

1) Der Hohmannit ist nach der Beschreibung, die Frenzel (Min. Milth. 9, 397 und 11, 215) von diesem Minerale giebt, zweifellos ein Zersetzungsproduct des A ma- 
Beilr. zur Kennln. der chem, Conslit. u. der Genese der natuirl. Eisensulfate. I. 215

als Hohmannit in Rechnung, um die theoretische Zusammensetzung des Pulvers, dessen Formel dann

$$
126\left[\mathrm{FeSO}_{4}+\mathrm{aq}\right]+\left[\mathrm{Fe}_{4} \mathrm{~S}_{3} \mathrm{O}_{15}+13 \mathrm{aq}\right]
$$

lauten würde, ermitteln zu können. Dieser Mischung entsprechen die in Columne b. angeführten Zahlen.

Der Gewichtsverlust dieses Pulvers betrug:

$$
\begin{array}{cl}
\text { uber Schwefelsäure } & 0,08 \% \\
\text { bei } 80^{\circ} & 0,67 \\
-150 & 0,37
\end{array}
$$

also in toto $1,12 \%$. Wenn bei $150^{\circ}$ die Oxydation schon vollendet war, mutssten hierzu noch $4,52 \%$ als Aequivalent fur den aufgenommenen Sauerstoff hiozukommen, so dass sich der Gesammtverlust bis $150^{\circ}$ auf $5,64 \%$ stellen wurde. Nach den in $\S 1$ gemachten Beobachtungen darf man schliessen, dass die Verbindung $\mathrm{FeSO}_{4}+$ aq bis $150^{0}{ }_{3}^{1}$ Molekul, uber $1500 \frac{2}{3}$ Molekul Wasser verliert. Dem würde ein theoretischer Gewichtsverlust von $3,20 \%$ bis $150^{\circ}$ und von $6,78 \%$ uber $150^{\circ}$ entsprechen. Es scheint also, dass bei $150^{\circ}$ noch nicht alles Eisenoxydul in Oxyd ubergegangen war. Zudem werden die Verbältnisse auch durch die Beimengung des Hohmannit-ähnlichen Sulfates verschleiert, uber dessen Verhallen

\begin{tabular}{|c|c|c|c|c|}
\hline & a. & Mol.-Quol.: & b. & Beob. - Rechn. \\
\hline $\mathrm{FeO}$ & 38,96 & 0,541 & 38,92 & $+0,04$ \\
\hline $\mathrm{SO}_{3}$ & 43,28 & 0,541 & 43,25 & $+0,03$ \\
\hline \multirow[t]{2}{*}{$\mathrm{H}_{2} \mathrm{O}$} & 17,76 & \multirow[t]{2}{*}{0,987} & 17,83 & $-0,07$ \\
\hline & 100,00 & & 100,00 & \\
\hline
\end{tabular}
beim Erhitzen noch gar nichts bekannt ist.

c) Die im Messingsiebe zurückgebliebenen härteren Theile hatten nachstehende Zusammensetzung:

Der Gewichtsverlust der centralen Partien war:

$$
\begin{aligned}
\text { über Schwefelsăure } & =0,62 \% \\
\text { bei } 80^{\circ} & =6,51 \\
-150 & =0,77
\end{aligned}
$$

Die Gewichtszunahme in Folge der Oxydation belrägt 4,33\%. Der Gesammtverlust von $80^{\circ}-150^{\circ}$ ist somit gleich $11,61 \%$. Es entfallen dann

rantites. Beide Mineralien lassen sich sehr leicht in eine chemische Beziebung zu einander bringen. Es sind drei Gleichungen möglich: Entweder erfolgt die Zersetzung des Amarantites unter Zutritt von Wasser nach der Gleichung: $2 \mathrm{Fe}_{2} \mathrm{~S}_{2} \mathrm{O}_{9}+\mathrm{H}_{2} \mathrm{O}=$ $\mathrm{Fe}_{4} \mathrm{~S}_{3} \mathrm{O}_{15}+\mathrm{H}_{2} \mathrm{SO}_{4}$ oder nach der Gleichung: $3 \mathrm{Fe}_{2} \mathrm{~S}_{2} \mathrm{O}_{9}=\mathrm{Fe}_{4} \mathrm{~S}_{3} \mathrm{O}_{25}+\mathrm{Fe}_{2} \mathrm{~S}_{3} \mathrm{O}_{12}$, oder endlich nach der Gleichung: $4 \mathrm{Fe}_{2} \mathrm{~S}_{2} \mathrm{O}_{9}=\mathrm{Fe}_{4} \mathrm{~S}_{3} \mathrm{O}_{15}+\mathrm{Fe}_{4} \mathrm{~S}_{5} \mathrm{O}_{21}$. Ich halte die letztere Gleichung auf Grund spater zu besprechender Beobachtungen für die wahrscheinlichere. Der Hohmannit ist nach Frenzel im Wasser unlöslich, dagegen ist die Verbindung $\mathrm{Fe}_{4} \mathrm{~S}_{5} \mathrm{O}_{21}$ löslich. Ibr wären dann die braunen Flecken zuzuschreiben, die beim Verstäuben von Eisenvitriol sehr häufig auf einer papiernen Unterlage entstehen. 
auf den Gewichtsverlust über $150^{\circ}$ nur $5,53 \%$. Die relativ grosse Gewichlsabnahme des Pulvers über Schwefelsäure macht es wahrscheinlich, dass neben den Verbindungen $\mathrm{FeSO}_{4}+$ aq und $\mathrm{FeSO}_{4}+4$ aq auch noch kleine Mengen von unverstäublem Eisenvitriol dem Pulver beigemengt waren. Die Constilution des Kernes kann durch die Formel :

$$
9\left[\mathrm{FeSO}_{4}+7 \mathrm{aq}\right]+96\left[\mathrm{FeSO}_{4}+4 \mathrm{aq}\right]+306\left[\mathrm{FeSO}_{4}+\mathrm{aq}\right]
$$

ausgedruckt werden. Die dieser Formel entsprechenden Zahlen sind oben in der Columne b. aufgefuhrt.

Der theoretische Wasserverlust bei verschiedenen Temperaturen berechnet sich nach obiger Formel wie folgt:

Tabelle IV.

\begin{tabular}{|c|c|c|c|c|c|c|}
\hline \multirow{4}{*}{$\begin{array}{c}\text { über Schwefels. } \\
\text { bei } 80^{\circ} \\
-150\end{array}$} & $9\left[\mathrm{FeSO}_{4}+7 \mathrm{aq}\right]$ & $96\left[\mathrm{FeSO}_{4}+4 \mathrm{aq}\right]$ & $306\left[\mathrm{FeSO}_{4}+\mathrm{aq}\right]$ & $\begin{array}{c}\text { Sum- } \\
\text { me }\end{array}$ & $\begin{array}{c}\text { Berechnet } \\
\mathrm{H}_{2} \mathrm{O} \%\end{array}$ & \multirow{2}{*}{ Beob. } \\
\hline & 27 Mol. & - & - & 27 & 0,64 & \\
\hline & $27-$ & $288 \mathrm{Mol}$. & - & 315 & 7,46 & \\
\hline & $3-$ & $32-$ & 102 Mol. & 137 & $3,24\}^{10,70}$ & 11,61 \\
\hline bis 300 & $1,5-$ & $16-$ & $51-$ & 68,5 & $1,62 \mid$ & 5.9 \\
\hline iber 300 & $4,5-$ & $48-$ & $153-$ & 205,5 & $4,87\}$ & 5,53 \\
\hline & 63,0 & 384 & 306 & 753 & 17,83 & 17,76 \\
\hline
\end{tabular}

Die Uebereinstimmung zwischen Beobachtung und Rechnung muss als befriedigend angesehen werden, weil ja nicht festgestellt ist, ob die Annahme, dass bei $150^{\circ}$ schon alles Eisenoxydul oxydirt sei, der Wirklichkeil entspricht. Hätle sich nur der fünfte Theil des Eisenoxyduls der Oxydation entzogen, so würde die Uebereinstimmung eine vollkommene sein.

Durch diese Versuche wird bewiesen, dass der Verstäubungsprocess des Eisenvitiols nur auf die Bildung von $\mathrm{FeSO}_{4}+$ aq hinarbeilet, und dass neben diesem Vorgange diegleichzeitig eintretende Oxydation als unwesentlich bezeichnet werden muss.

Die vollkommene Umwandlung eines Eisenvitriolkrystalles in $\mathrm{FeSO}_{4}$ +aq nimmt eine ziemlich lange Zeit in Anspruch. Die drei ersten Krystallwassermoleküle entweichen auch beim Liegen an der Luft sehr rasch, je nach der Grösse der angewendeten Krystalle in 24 Stunden bis 3 Wochen. Dann nimmt aber das Gewicht sehr langsam und gegen Ende durch viele Wochen kaum merklich ab. Erst nach Jahren ist der Krystall vollends in $\mathrm{FeSO}_{4}+$ aq umgewandelt. Zwei Krystalle, welche vier Jahre der Einwirkung der Zimmerluft ausgesetzt waren, hatten nachstehende Zusammenselzung: 
Beilr. zur Kenntn. der chem. Constil. u. der Genese der natïrl. Eisensulfate. I. 217

\begin{tabular}{lrcrc} 
& a. & Mol.-Quot.: & \multicolumn{1}{c}{ b. } & Mol.-Quot.: \\
$\mathrm{FeO}$ & 41,34 & 0,574 & 40,75 & 0,566 \\
$\mathrm{Fe}_{2} \mathrm{O}_{3}$ & 0,05 & - & 0,52 & 0,003 \\
$\mathrm{SO}_{3}$ & 46,34 & 0,579 & 46,61 & 0,583 \\
$\mathrm{H}_{2} \mathrm{O}$ & 12,27 & 0,682 & $\frac{12,12}{100,00}$ & 0,673
\end{tabular}

Bei beiden Krystallen ist das Verbältniss von $\mathrm{SO}_{3}: \mathrm{H}_{2} \mathrm{O}=1: 1,17$. Die geringe Bedeutung der bei der Verstäubung eintretenden Oxydation wird durch diese Analysen gleichfalls bewiesen.

\section{Die Oxydation von Eisenvitriollösungen.}

Wenn eine Eisenvitriollösung längere Zeil der Einwirkung des almosphärischen Sauerstoffes ausgesetzt wird, so nimmt dieselbe unter gleichzeitiger Abscheidung eines gelbbraunen Niederschlages eine braunrolhe Färbung an. Sowohl die Zusammensetzung des Niederschlages als auch jene der Lösung wechselt je nach dem jeweiligen Concentrationsgrade der Lösung. Eine Gesetzmässigkeit konnte bisher nicht aufgefunden werden wahrscheinlich deshalb, weil auf zwei Umstände, welche den glatten Verlauf der Reactionen verschleiern, nicht genugend Rücksicht genommen wurde :

1) scheint man ubersehen zu haben, dass der gelbbraune Niederschlag von basischem Ferrisulfat beim lange fortgesetzten Waschen mit Wasser bestandig Schwefelsäure an das Waschwasser abgiebt, wodurch im Niederschlage das Molekularverbältniss $\mathrm{Fe}_{2} \mathrm{O}_{3}: \mathrm{SO}_{3}$ stets zu Ungunsten der Schwefelsäure beeinflusst wird;

2) dürfte es nicht genugend beachtet worden sein, dass sich in wässerigen Lösungen nicht allein das directe Oxydationsproduct des Eisenvitriols, die Verbindung $\mathrm{Fe}_{2} \mathrm{~S}_{2} \mathrm{O}_{9}$, zerselzt, sondern dass auch das normale Ferrisulfat $\mathrm{Fe}_{2} \mathrm{~S}_{3} \mathrm{O}_{12}$ - selbst ohne Erbitzen - einer ähnlichen Zerselzung anheimfällt, wenn dessen Lösung uber eine gewisse Grenze hinaus verdünnt wird.

Bevor also auf die Oxydation von Eisenvitriollösungen selbst eingegangen werden kann, muss diese Erscheinung näher untersucht werden.

§ 3. Die Zersetzung einer verdunnten Lösung von normalem Ferrisulfat. Die Ferrisulfatlösung, welche zu den vachstehenden Versuchen verwendet wurde, enthielt im Cubikcentimeter im Mitlel ·

$$
\begin{array}{ll}
\mathrm{Fe}_{2} \mathrm{O}_{3} & 0,0672 \mathrm{~g} \\
\mathrm{SO}_{3} & 0,1013 \mathrm{~g} \\
\cline { 2 - 2 } & 0,1685 \mathrm{~g}
\end{array}
$$

Das Molekularverhältniss $\mathrm{Fe}_{2} \mathrm{O}_{3}: \mathrm{SO}_{3}=1: 3,01$. Die Lösung war in der vorliegenden Concentration gelbroth. Eine merkliche Trubung trat erst ein, als $1 \mathrm{ccm}$ der Lösung mit $89 \mathrm{~cm} \mathrm{H}_{2} \mathrm{O}$ verdunnt worden war. Die Verdunnung, d. h. die Zahl der Cubikcentimeter Wasser, in denen $0,1 \mathrm{~g}$ Salz gelöst war, betrug 53,4 . 
Die Versuche wurden so ausgeführt, dass nach 24 Stunden beziehungsweise 8 Tagen der Niederschlag auf einem kleinen Filter gesammell wurde. Das Filtrat, dem kein Tropfen Waschwasser beigemengt war, der Niederschlag, der bis zum Verschwinden der Eisenoxydreaction gewaschen wurde, und das Waschwasser wurden jedes für sich analysirt.

Wie die nachstehende Tabelle $\mathrm{V}$ beweist, ist das Molekularverhältniss von $\mathrm{Fe}_{2} \mathrm{O}_{3}: \mathrm{SO}_{3}$ im Filtrat sowohl von der Concentration als auch von der Zeit, welche zwischen Verdünnung und Filtration verfloss, abbängig. Der Exponent dieses Verhältnisses ist um so grösser, je verdunnter die Lösung war, und bei gleicher Verdínnung um so grösser, je mehr Zeit zwischen dem Momente der Verdunnung und dem der Filtration verstrich.

Tabelle V.

\begin{tabular}{|c|c|c|c|c|c|c|}
\hline \multirow{3}{*}{\multicolumn{3}{|c|}{$\begin{array}{c}1 \\
1 \text { ccm Lösung } \\
\text { verdünnt mit }\end{array}$}} & \multicolumn{4}{|c|}{ Abfiltrirt wurde nach } \\
\hline & & & \multicolumn{2}{|c|}{24 Stunden } & \multicolumn{2}{|l|}{8 Tagen } \\
\hline & & & $\begin{array}{l}\text { Im Filtrat waren } \\
\text { enthalten }\end{array}$ & $\frac{\mathrm{SO}_{3}}{\mathrm{Fe}_{2} \mathrm{O}_{3}}$ & $\begin{array}{l}\text { Im Filtrat waren } \\
\text { enthalten }\end{array}$ & $\frac{\mathrm{SO}_{3}}{\mathrm{Fe}_{2} \mathrm{O}_{3}}$ \\
\hline \multicolumn{3}{|c|}{$89 \mathrm{ccm} \mathrm{H}_{2} \mathrm{O}$} & & & $\begin{aligned} \mathrm{Fe}_{2} \mathrm{O}_{3} & =0,0624 \\
\mathrm{SO}_{3} & =0,0989\end{aligned}$ & 3,17 \\
\hline 100 & - & - & & & $\begin{aligned} \mathrm{Fe}_{2} \mathrm{O}_{3} & =0,05721 \mathrm{j} \\
S \mathrm{O}_{3} & =0,0957\end{aligned}$ & 3,35 \\
\hline 200 & $\overrightarrow{-}$ & - & $\begin{array}{r}\mathrm{Fe}_{2} \mathrm{O}_{3}=0,0619 \\
\mathrm{SO}_{3}=0,1021\end{array}$ & 3,29 & $\begin{array}{r}\mathrm{Fe}_{2} \mathrm{O}_{3}=0,0431 \\
\mathrm{SO}_{3}=0,0917\end{array}$ & 4,25 \\
\hline 300 & - & - & $\begin{array}{r}\mathrm{Fe}_{2} \mathrm{O}_{3}=0,0509 \\
S \mathrm{SO}_{3}=0,0913\end{array}$ & 3,58 & & \\
\hline 400 & - & - & $\begin{aligned} \mathrm{Fe}_{2} \mathrm{O}_{3} & =0,0414 \\
\mathrm{SO}_{3} & =0,0960\end{aligned}$ & $\left.4,64^{2}\right)$ & $\begin{aligned} \mathrm{Fe}_{2} \mathrm{O}_{3} & =0,0298 \\
\mathrm{SO} O_{3} & =0,0912\end{aligned}$ & 6,12 \\
\hline 600 & - & - & $\begin{array}{r}\mathrm{Fe}_{2} \mathrm{O}_{3}=0,0300 \\
\mathrm{SO}_{3}=0,0912\end{array}$ & $6,083)$ & $\begin{array}{r}\mathrm{Fe}_{2} \mathrm{O}_{3}=0,0224 \\
\mathrm{SO}_{3}=0,0932\end{array}$ & 8,32 \\
\hline 800 & - & - & $\begin{aligned} \mathrm{Fe}_{2} \mathrm{O}_{3} & =0,0258 \\
S O_{3} & =0,0914\end{aligned}$ & $\left.7,10^{4}\right)$ & $\begin{aligned} \mathrm{Fe}_{2} \mathrm{O}_{3} & =0,0167 \\
\mathrm{SO}_{3} & =0,0905\end{aligned}$ & 10,78 \\
\hline 1000 & - & - & $\begin{array}{r}\mathrm{Fe}_{2} \mathrm{O}_{3}=0,0212 \\
\mathrm{SO}_{3}=0,0896\end{array}$ & 8,45 & $\begin{array}{r}\mathrm{Fe}_{2} \mathrm{O}_{3}=0,0119 \\
\mathrm{SO}_{3}=0,0887\end{array}$ & 14,78 \\
\hline 1200 & - & - & $\begin{aligned} \mathrm{Fe}_{2} \mathrm{O}_{3} & =0,0178 \\
S O_{3} & =0,0921\end{aligned}$ & 10,35 & $\begin{aligned} \mathrm{Fe}_{2} \mathrm{O}_{3} & =0,0108 \\
\mathrm{SO}_{3} & =0,0909\end{aligned}$ & 16,83 \\
\hline 1400 & - & - & $\begin{aligned} \mathrm{Fe}_{2} \mathrm{O}_{3} & =0,0134 \\
S O_{3} & =0,0900\end{aligned}$ & 13,44 & $\begin{array}{r}\mathrm{Fe}_{2} \mathrm{O}_{3}=0,0089 \\
\mathrm{~S} \mathrm{O}_{3}=0,0948\end{array}$ & 21,07 \\
\hline 2000 & - & - & $\begin{aligned} \mathrm{Fe}_{2} \mathrm{O}_{3} & =0,0090 \\
\mathrm{SO}_{3} & =0,0905\end{aligned}$ & 20,11 & $\begin{aligned} \mathrm{Fe}_{2} \mathrm{O}_{3} & =0,0061 \\
\mathrm{SO}_{3} & =0,0924\end{aligned}$ & 30,80 \\
\hline 2800 & - & - & $\begin{aligned} \mathrm{Fe}_{2} \mathrm{O}_{3} & =0,0064 \\
\mathrm{~S} O_{3} & =0,0903\end{aligned}$ & 28,22 & & \\
\hline 3000 & - & - & & & $\begin{aligned} \mathrm{Fe}_{2} \mathrm{O}_{3} & =0,0051 \\
\mathrm{SO}_{3} & =0,0925\end{aligned}$ & 36,28 \\
\hline 4000 & - & - & $\begin{aligned} \mathrm{Fe}_{2} \mathrm{O}_{3} & =0,0042 \\
S O_{3} & =0,0915\end{aligned}$ & 43,57 & $\begin{array}{r}\mathrm{Fe}_{2} \mathrm{O}_{3}=0,0040 \\
\mathrm{SO}_{3}=0,092 .\end{array}$ & 46,05 \\
\hline
\end{tabular}

1) Der Niederschlag wurde nach 3 Wochen abfiltrirt.

2) Ein zweiter Versuch ergab das Verhältniss $\mathrm{Fe}_{2} \mathrm{O}_{3}: \mathrm{SO}_{3}=1: 4,53$.

3) - $\quad$ - $\quad$ - $\quad$ - $\quad$ - $\quad$ - $\quad$ - $=1: 5,90$.

4) Zwei weitere Versuche ergaben das Verhältniss von $\mathrm{Fe}_{2} \mathrm{O}_{3}: \mathrm{SO}_{3}=1: 6,92$ resp. 7, 15 . 
Diese Erscheinung erklärt sich dadurch, dass mit zunehmender Verdünnung immer grössere Mengen eines basischen Eisensulfales herausfallen. Denn nicht allein der Eisen-, sondern auch der Schwefelsäuregehalt der Lösung vermindert sich beständig sowohl bei 24 stündiger, als auch bei 8 tägiger Reactionsdauer mit zunehmender Verdünnung.

Tabelle VI.

\begin{tabular}{|c|c|c|c|c|c|c|c|c|c|}
\hline $\begin{array}{l}1 \mathrm{ccm} \text { Lösung wurde } \\
\text { verdünnt mit } x \mathrm{ccm} \\
\qquad \mathrm{H}_{2} \mathrm{O}\end{array}$ & $\begin{array}{c}x= \\
200\end{array}$ & $\begin{array}{l}x= \\
400\end{array}$ & $\begin{array}{c}x= \\
600\end{array}$ & $\begin{array}{l}x= \\
800\end{array}$ & $\begin{array}{l}x= \\
1000\end{array}$ & $\begin{array}{l}x= \\
1200\end{array}$ & $\begin{array}{l}x= \\
1400\end{array}$ & $\begin{array}{l}x= \\
2000\end{array}$ & $\begin{array}{l}x= \\
4000\end{array}$ \\
\hline $\begin{array}{l}\text { nach } 24 \text { Stunden wa- } \\
\text { ren statt } 40 \% \mathrm{Fe}_{2} \mathrm{O}_{3} \\
\text { noch in Lösung ... } \\
\text { nach } 8 \text { Tagen waren } \\
\text { statt } 40 \% \mathrm{Fe}_{2} \mathrm{O}_{3} \text { noch } \\
\text { in Lösung . . . . }\end{array}$ & $\begin{array}{c}\% \\
36,34\end{array}$ & $\begin{array}{c}0 / 0 \\
26,17\end{array}$ & $\begin{array}{c}\% \\
18,47\end{array}$ & $\begin{array}{c}\% \\
15,78\end{array}$ & $\begin{array}{r}\% \\
12,78 \\
7,50\end{array}$ & $\begin{array}{c}\% \\
10,49\end{array}$ & $\begin{array}{l}\% \\
8,06\end{array}$ & $\begin{array}{l}\% \\
5,39\end{array}$ & 2,46 \\
\hline $\begin{array}{l}\text { nach } 24 \text { Stunden wa- } \\
\text { ren statt } 60 \% \mathrm{SO}_{3} \\
\text { noch in Lösung. . } \\
\text { nach } 8 \text { Tagen waren } \\
\text { statt } 60 \% \mathrm{SO}_{3} \text { noch } \\
\text { in Lösung . . . . }\end{array}$ & 59,85 & 56,30 & 56,14 & 55,79 & 55,01 & 54,27 & 54,21 & 53,68 & 53,57 \\
\hline
\end{tabular}

Dies erhellt aus Tabelle VI. Dieselbe zeigt, wie viel Procente Eisenoxyd beziehungsweise Schwefelsäure anstatt der $40 \% \mathrm{Fe}_{2} \mathrm{O}_{3}$ resp. $60 \%$ $\mathrm{SO}_{3}$ des unzersetzten Salzes nach 24 stündiger bez. 8 tägiger Reactionsdauer noch in der Lösung enthalten waren. Aus derselben Tabelle geht ferner auch hervor, dass die absolute Menge des gefällten Eisensulfates nach 8 tågiger Reactionsdauer stets grösser ist als nach 24 sttindiger. Die Differenz zwischen beiden Versuchsreihen wird mit steigender Verdunnung immer kleiner. Sie beträgt $9,12 \% \mathrm{Fe}_{2} \mathrm{O}_{3}$ und $1,94 \% \mathrm{SO}_{3}$, wenn $1 \mathrm{ccm}$ Lösung mit $200 \mathrm{ccm} \mathrm{H}_{2} \mathrm{O}$ verdunnt wurde, dagegen nur mehr $0,09 \% \mathrm{Fe}_{2} \mathrm{O}_{3}$ und $0,26 \% \mathrm{SO}_{3}$, wenn $4000 \mathrm{ccm} \mathrm{H}_{2} \mathrm{O}$ zu $1 \mathrm{ccm}$ Lösung gegeben wurden. Die Ausfällung erreicht somit um so rascher ihr Ende, je stärker die ursprüngliche Lösung verdünnt wird.

Die chemische Zusammenselzung des Niederschlages entspricht der Formel $\mathrm{Fe}_{6} \mathrm{SO}_{12}$. (Vergl. Tabelle VII.) Die Differenzen, welche zwischen Beobachtung und Rechnung bestehen, sind sebr gering. Sie betragen bei der angewandten Menge nur 0,0002 $\mathrm{g}$ im Miltel: Mit diesem Ergebnisse stimmen auch die Beobachtungen $\mathbf{S}$ cheerer's ${ }^{1}$ ) uberein, der beim Kochen einer verdunnten Lösung von normalem Ferrisulfat einen ganz analogen Niederschlag erhielt.

1) Pogg. Ann. 1838, 44, 453. 
Die Zersetzung, welche wahrscheinlich nach der Gleichung:

$$
3\left[\mathrm{Fe}_{2} \mathrm{~S}_{3} \mathrm{O}_{12}\right]+8 \mathrm{aq}=\mathrm{Fe}_{6} \mathrm{SO}_{12}+8 \mathrm{H}_{2} \mathrm{SO}_{4}
$$

verläuft, lässt sich durch eine einfache Curve graphisch darstellen, wenn man auf die Ordinatenaxe die Procente des ausgefăliten Eisenoxydes den Gesammtgehalt der Lösung an Eisenoxyd $=100 \%$ geselzt - und auf die Abcissenaxe die Verdunnung, d. i. die Menge Wasser, in der $0,1 \mathrm{~g}$ $\mathrm{Fe}_{2} \mathrm{~S}_{3} \mathrm{O}_{12}$ gelöst war, aufträgt. (Vergl. Tabelle VII.)

Tabelle VII.

\begin{tabular}{|c|c|c|c|c|}
\hline \multicolumn{2}{|c|}{$\begin{array}{l}0,1 \mathrm{~g} \mathrm{Fe}\left(\mathrm{SO}_{4}\right)_{3} \\
\text { ist gelöst in }\end{array}$} & $\begin{array}{c}\text { Ausgefälltes } \mathrm{Fe}_{2} \mathrm{O}_{3} \\
\text { in } \% \text { des Gesammt- } \\
\text { eisengehaltes } \\
\end{array}$ & $\begin{array}{c}\text { In den Nieder- } \\
\text { schlag überge- } \\
\text { gangene Schwefel- } \\
\text { saure }\end{array}$ & $\frac{F e_{2} O_{3}}{S O_{3}}$ \\
\hline \multicolumn{2}{|c|}{$52,4 \mathrm{ccm} \mathrm{H}_{2} \mathrm{O}$} & $8,71 \%$ & 0,590 & 3,05 \\
\hline 62,8 & $-\quad \cdot-$ & 9,75 & 0,74 & 2,58 \\
\hline 124,6 & $-\quad-$ & 31,62 & 2,29 & 2,83 \\
\hline 243,2 & $-\quad-$ & 54,03 & 3,70 & 2,91 \\
\hline 350,8 & $-\quad-$ & 66,67 & 4,57 & 2,92 \\
\hline 473,1 & - & 74,89 & 5,01 & 3,00 \\
\hline 600,8 & - & 81,06 & 5,40 & 2,98 \\
\hline 703,2 & - & 84,50 & 5,80 & 3,00 \\
\hline 787,1 & - & 87,50 & 5,84 & 3,00 \\
\hline 1158,7 & - & 90,87 & 5,90 & 3,07 \\
\hline 1761,8 & - & 92,43 & 5,17 & $\mathbf{3 , 5 5}$ \\
\hline 2374,4 & - & 93,99 & 5, 17 & 3,55 \\
\hline
\end{tabular}

Die Curve, welche bei einer Verdünnung von circa 48 beginnt, steigt anfangs stehr steil an und nähert sich im weiteren Verlaufe immer mehr

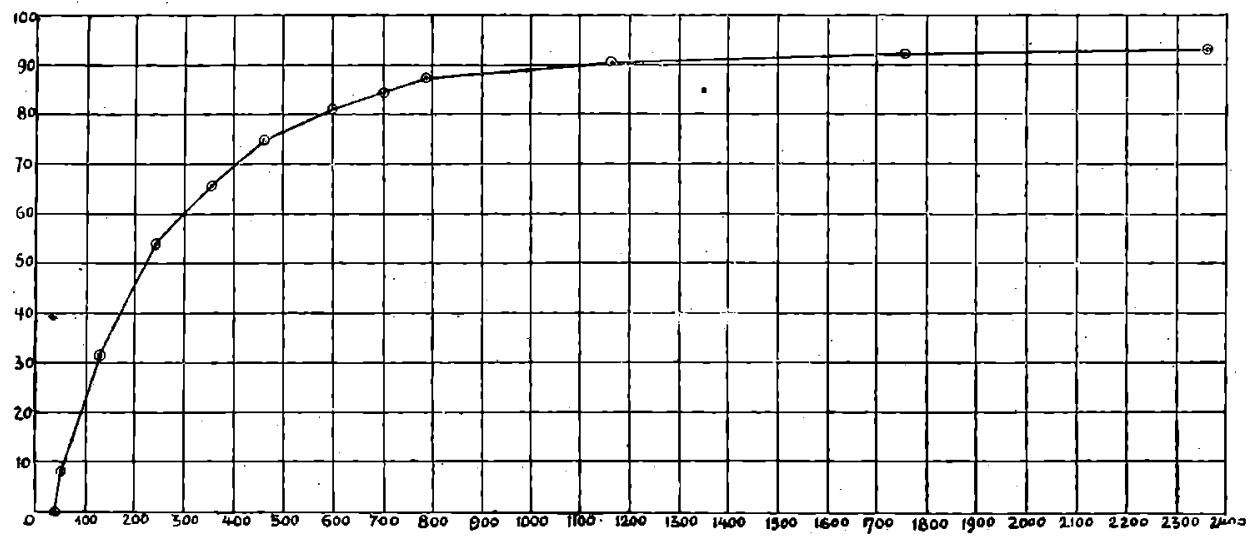

einer Parallelen zur Abeissenaxe, mit welcher sie vollkómmen parallel läuft, wenn $y=100 \%$ ist, d. h. wenn alles Eisenoxyd ausgefällt ist. Dann. 
ändert natürlich eine weitere Verdünnung der Lösung nichls mehr an der Menge des Niederschlages. Den Verdünnungsgrad, bei welchem alles Eisenoxyd ausgefällt wird, konnte ich durch das Experiment nicht feststellen, weil die zur Verdunnung nothwendigen Wassermengen zu bedeutend geworden wären.

Vergleicht man die Resultate der Scheerer'schen Untersuchungen mit den obigen Ergebnissen, so ersieht man, wie sehr durch Erhitzen der Lösung die Zersetzung gefördert wird. Scheerer giebt namlich an, dass

bei einer Verdunnung von $1 \mathrm{~g} \mathrm{Fe}_{2} \mathrm{~S}_{3} \mathrm{O}_{12}$ auf $200 \mathrm{ccm} \mathrm{H}_{2} \mathrm{O}$ ungefähr die Hälfte des Gesammteisengehaltes,

bei einer Verdunnung von $1 \mathrm{~g} \mathrm{Fe}_{2} \mathrm{~S}_{3} \mathrm{O}_{12}$ auf $400 \mathrm{ccm} \mathrm{H}_{2} \mathrm{O}$ ungefähr $\frac{3}{4}$ des Gesammteisengebaltes,

bei einer Verdunnung von $1 \mathrm{~g} \mathrm{Fe}_{2} \mathrm{~S}_{3} \mathrm{O}_{12}$ auf $800 \mathrm{ccm} \mathrm{H}_{2} \mathrm{O}$ ungefähr $\frac{7}{8}$ des Gesammteisengehaltes,

bei einer Verdunnung von $1 \mathrm{~g} \mathrm{Fe}_{2} \mathrm{~S}_{3} \mathrm{O}_{12}$ auf $1000 \mathrm{ccm} \mathrm{H}_{2} \mathrm{O}$ ungefähr $\frac{9}{10}$ des Gesammteisengehaltes

herausfalle, welche Wirkung nach meinen Untersuchungen ohne Anwendung von Wärme erst bei Verdünnungen von $1 \mathrm{~g} \mathrm{Fe}_{2} \mathrm{~S}_{3} \mathrm{O}_{12}$ in circa 2000, 5000,7871 und $11587 \mathrm{ccm}$ Wasser erreicht wirde.

Sowohl die in Tabelle VII mitgetheilten Werthe für den Exponenten des Molekularverbältnisses $\mathrm{Fe}_{2} \mathrm{O}_{3}: \mathrm{SO}_{3}$, als auch aus den in den Niederschlag ubergegangenen Schwefelsäuremengen geht hervor, dass bei einer $1158,7 \mathrm{ccm}$ uberschreitenden Verdunnung die Niederschläge schwefelsäurearmer werden. Es gewinnt daher den Anschein, als ob grosse Mengen von Wasser dem Niederschlage von der Zusammensetzung $\mathrm{Fe}_{6} \mathrm{SO}_{12}$ Schwefelsäure zu entziehen vermöchten.

§ 4. Die Zersetzung oxydirter Eisenvilriollösungen. Die eben milgetheilten Thatsachen mussen berucksichtigt werden, wenn man sich Klarheit daruber verschaffen will, wie der Oxydationsprocess einer verdünnten Eisenvitriollösung verläuft. Bekanntlich bestehen darüber drei Ansichten:

1) Gmelin stellt in seinem Lehrbuche ${ }^{1)}$ fur den Oxydations- und Zerselzungsvorgang die Gleichung: $10 \mathrm{FeSO}_{4}+5 \mathrm{O}=\mathrm{Fe}_{4} \mathrm{SO}_{9}+3 \mathrm{Fe}_{2} \mathrm{~S}_{3} \mathrm{O}_{12}$ auf.

2) Nach Wittstein ${ }^{2}$ ) verläuft dieser Vorgang wie folgt: $6 \mathrm{FeSO}_{4}+$ $3 \mathrm{O}=\mathrm{Fe}_{4} \mathrm{~S}_{3} \mathrm{O}_{15}+\mathrm{Fe}_{2} \mathrm{~S}_{3} \mathrm{O}_{12}$.

3) Berzelius ${ }^{3}$ ) endlich nimmt an, dass sich eine oxydirte Eisenvitriollösung nach der Gleichung: $4 \mathrm{FeSO}_{4}+2 \mathrm{O}=\mathrm{Fe}_{2} \mathrm{SO}_{6}+\mathrm{Fe}_{2} \mathrm{~S}_{3} \mathrm{O}_{12}$ zerlege.

1). Handbuch der anorg. Chemie 6. Aufl., 3, 342 und 345.

2) Buchner, Rep. 3. Reihe, 1. Heft 2.

3) Lehrbuch der anorganischen Chemie 5. Aufl., 3, 596. Ich entnebme diese Literalurcilate der Arbeil M u ck's (Journ. f. prakt. Chemie 99, 403). 
So verschieden auch im Grossen und Ganzen die Ansichten der drei Chemiker sind, in Einem stimmen alle überein, nämlich dass schliesslich normales Eisenoxydsulfat in der Lösung enthalten sei.

Bei der Oxydation und Zersetzung von $1 \mathrm{~g}$ Eisenvitriol würde im Niederschlage beziehungsweise in der Lösung enthalten sein :

Tabelle VIII.

\begin{tabular}{|c|c|c|c|}
\hline $\begin{array}{c}\text { Wenn die Zersetzung } \\
\text { verltiuft }\end{array}$ & & Niederschlag & Lösung \\
\hline \multirow[t]{2}{*}{ nach Gleichung I } & $\begin{array}{l}\mathrm{Fe}_{2} \mathrm{O}_{3} \\
\mathrm{SO}_{3}\end{array}$ & $\begin{array}{l}0,1151 \mathrm{~g} \\
0,0288\end{array}$ & $\begin{array}{l}0,1727 \mathrm{~g} \\
0,2590\end{array}$ \\
\hline & & $0,1439 \mathrm{~g}$ & $0,4317 \mathrm{~g}$ \\
\hline \multirow[t]{2}{*}{ nach Gleichung II } & $\begin{array}{l}\mathrm{Fe}_{2} \mathrm{O}_{3} \\
\mathrm{SO}_{3}\end{array}$ & $\begin{array}{l}0,1919 \mathrm{~g} \\
0,4439\end{array}$ & $\begin{array}{l}0,0959 \mathrm{~g} \\
0,1439\end{array}$ \\
\hline & & $0,3358 \mathrm{~g}$ & $0,2398 \mathrm{~g}$ \\
\hline \multirow[t]{2}{*}{ nach Gleichung III } & $\begin{array}{l}\mathrm{Fe}_{2} \mathrm{O}_{3} \\
\mathrm{SO}_{3}\end{array}$ & $\begin{array}{l}0,1439 \mathrm{~g} \\
0,0720\end{array}$ & $\begin{array}{l}0,1439 \mathrm{~g} \\
0,2158\end{array}$ \\
\hline & & $0,2159 \mathrm{~g}$ & $0,3597 \mathrm{~g}$ \\
\hline
\end{tabular}

Um nun die im vorigen Paragraphen festgestellte Verdúnnungsgrenze für normale Ferrisulfatlösungen, d. i. $1 \mathrm{~g} \mathrm{Fe}_{2} \mathrm{~S}_{3} \mathrm{O}_{12}$ in circa $500 \mathrm{ccm}$ Wasser, nicht zu uberschreiten, soll $1 \mathrm{~g}$ Eisenvitriol, falls die Reaction nach

Gleichung I verläuft, in höchstens $215,9 \mathrm{ccm}$

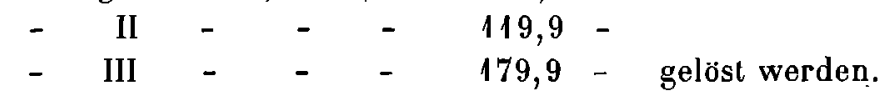

Die im Nachstehenden mitgetheilten Versuche wurden so ausgefuhrt, dass stets $1 \mathrm{~g}$ Eisenvitriol mit Wasserstoffsuperoxyd oxydirt wurde, damit mit einem Schlage das ganze Eisenoxydul in Oxyd ibergeführt werde. Dann blieb die Lösung 12 Stunden, beziehungsweise 8 Tage stehen. Der flüssige Autheil wurde durch Filtration von dem Niederschlage getrennt und, ohne dass ein Tropfen Waschwasser beigemengt wurde, anajysirt. Der Niederschlag wurde bis zum Verschwinden der Eisenreaction gewaschen 1). Die directen Analysenergebnisse sind in nachstehender Tabelle aufgefthrt.

1) Muck, der im Jahre 1866 (Journ. f. prakt. Chemie 99, 103) ähnliche Versuche ausführte, liess Eisenvitriollösungen von beslimmler Concentration langsam an der Luft oxydiren. Diese Methode hat, abgesehen davon, dass sic sehr viel Zeit in Anspruch nimmt, noch den Uebelstand, dass man nie eindeulige Resultate erhält, weil sich nie alles Eisenoxydul freiwillig oxydirt. Auch ist es fast unmöglich, die Lösung auf einem constanten Concentrationspunkte zu erhalten. 
Beitr. zur Kenntn. der chem. Constit. u. der Genese der nalürl. Eisensulfate. I. 223

Tabelle IX.

\begin{tabular}{|c|c|c|c|c|c|c|c|c|c|c|c|}
\hline & \multirow{2}{*}{\multicolumn{3}{|c|}{$\begin{array}{c}1 \mathrm{~g} \mathrm{FeSO}_{4}+ \\
7 \text { aq wurde } \\
\text { gelöst in }\end{array}$}} & \multirow{2}{*}{ Es enthielten: } & \multicolumn{3}{|c|}{ Nach 12 Stunden } & \multicolumn{4}{|c|}{ Nach 8 Tagen } \\
\hline & & & & & $\mathrm{Fe}_{2} \mathrm{O}_{3}$ & $\mathrm{SO}_{3}$ & $\mathrm{Fe}_{2} \mathrm{O}_{3}: \mathrm{SO}_{3}$ & $\mathrm{Fe}_{2} \mathrm{O}_{3}$ & $\mathrm{SO}_{3}$ & $\mathrm{Fe}_{2} \mathrm{O}$ & ${ }_{3}: \mathrm{SO}_{3}$ \\
\hline \multirow{3}{*}{1} & & & & Filtrat & 0,1800 & 0,2379 & $: 2,64$ & $|0,1727|$ & 0,2356 & 1 & 2,73 \\
\hline & $12 \frac{1}{2} \mathrm{c} C \mathrm{C}$ & $\operatorname{ccm}$ & $\mathrm{H}_{2} \mathrm{O}$ & Niederschlag & 0,0881 & 0,0203 & $2,17: 1$ & 0,0923 & 0,0200 & 2,31 & 1 \\
\hline & & & & Waschwasser & 0,0185 & 0,0260 & $1: 2,80$ & 0,0211 & 0,0306 & 1 & 2,90 \\
\hline \multirow{3}{*}{2} & & & & Fillrat & $0, \mathbf{1 8} 17$ & 0,2442 & $: 2,69$ & 0,1695 & 0,2361 & 1 & $: 2,79$ \\
\hline & 25 & - & - & Niederschlag & $0, C 976$ & 0,0199 & $2,45: 1$ & 0,1052 & 0,0204 & 2,58 & $: 1$ \\
\hline & & & & Waschwasser & 0,0133 & 0,0200 & $1: 3,01$ & 0,0171 & 0,0273 & 1 & $: 3, \mathbf{2 0}$ \\
\hline \multirow{3}{*}{3} & & & & Filtrat & $0,17 \mathrm{s3}$ & 0,2465 & $1: 2,84$ & 0,1644 & 0,2450 & 1 & $: 2,98$ \\
\hline & 50 & - & - & Niederschlag & 0,1100 & 0,0233 & $2,36: 1$ & 0,1156 & 0,0233 & 2,48 & $: 1$ \\
\hline & & & & Waschwasser & 0,0079 & 0,0446 & $1: 3,70$ & 0,0086 & 0,0157 & 1 & $: 3,65$ \\
\hline \multirow{3}{*}{4} & & & & Filtrat & 0,1643 & 0,2453 & $: \mathbf{2 , 9 9}$ & $\mid 0,4597$ & 0,2475 & 1 & 3,10 \\
\hline & 75 & - & - & Niederschlag & 0,1199 & 0,0260 & $2,31: 1$ & 0,1257 & 0,0266 & 2,37 & $: 1$ \\
\hline & & & & Waschwasser & 0,0069 & 0,0132 & $1: 3,88$ & 0,0047 & 0,0114 & 1 & $: 4,85$ \\
\hline \multirow{3}{*}{5} & & & & Filtrat & 0,1633 & 0,2473 & $1: 3,03$ & $\mid 0,1515$ & 0,2491 & 1 & $: 3,29$ \\
\hline & 100 & - & - & Niederschlag & 0,1230 & 0,0271 & $2,27: 1$ & 0,1332 & 0,0278 & 2,39 & $: 1$ \\
\hline & & & & Waschwasser & 0,0050 & 0,0099 & $1: 3,96$ & 0,0040 & 0,0094 & 1 & 4,09 \\
\hline \multirow{3}{*}{6} & & & & Filtrat & 0,1544 & 0,2469 & $1 \quad: 3,20$ & $\mid 0,1 \mathbf{6} \mathbf{3} 8$ & 0,2476 & 1 & $: 3,44$ \\
\hline & 150 & - & - & Niederschlag & 0,1327 & 0,0298 & $2,22: 1$ & 0,1439 & 0,0299 & 2,41 & $: 1$ \\
\hline & & & & Waschwasser & 0,0036 & 0,0092 & $1: 5,11$ & 0,0033 & 0,0088 & 1 & $: \mathbf{5 , 3 3}$ \\
\hline \multirow{3}{*}{7} & & & & Filtrat. & 0,1486 & 0,2468 & $: 3,32$ & $\mid 0,1343$ & 0,2461 & 1 & 3,67 \\
\hline & 200 & - & - & Niederschlag & 0,1384 & 0,0304 & $2,28: 1$ & 0,1538 & 0,0315 & 2,44 & 1 \\
\hline & & & & Waschwasser & 0,0032 & 0,0077 & $1: 4,81$ & 0,0029 & 0,0078 & 1 & $: 5,26$ \\
\hline \multirow{3}{*}{8} & & & & Filtrat & 0,1403 & 0,2457 & $1: 3,50$ & 0,1291 & 0,2425 & 1 & 3,76 \\
\hline & 250 & - & - & Niederschlag & 0,1479 & 0,0312 & $2,37: 1$ & 0,1591 & 0,0325 & 2,45 & $: 1$ \\
\hline & & & & Waschwasser & $0 ; 0023$ & 0,0072 & $1: 6,26$ & 0,0021 & 0,0094 & 1 & : 8,95 \\
\hline
\end{tabular}

Aus dieser Zusammenslellung ergeben sich folgende Thatsachen:

1) Die Zusammensetzung der oxydirten Eisenvitriollösung ist eine Function der Verdúnnung und bei gleicher Verdunnung eine Function der Reactionsdauer. Der Exponent des Molekularverhältnisses $\mathrm{SO}_{2}: \mathrm{Fe}_{2} \mathrm{O}_{3}$ steht zu den beiden Factoren in einem geraden Verhältnisse. Er wächsı mit uunehmender Verdunnung und mit zunehmender Reactionsdauer. Nach achttägigem Stehen scheint das chemische Gleichgewicht zwischen Niederschlag und Lösung vollkommen hergestellt zu sein, denn, wie Controlversuche lehrten, bleibt die abfltrirte Lösung dann vollkommen klar.

2) Normales Eisenoxydsulfat ist vorübergehend nur in einer Lösung von $1 \mathrm{~g}$ Eisenvitriol auf $75 \mathrm{ccm}$ Wasser nach zwölfstündiger Reactionsdauer [Tabelle IX 4 und Tabelle $X \mathrm{a}$ ], dauernd bei einer Verdunnung von $1 \mathrm{~g}$ Éisenvitriol auf $50 \mathrm{ccm}$ Wasser nach achttägiger Reactionsdauer [Tabelle IX3 
und Tabelle X b] vorbanden. Bei grösserer Concentration enthält die Lösung ein basisches, bei geringerer Concentration anscheinend ein saures Salz.

3) In den beiden hier angegebenen Fallen verläuft der Zersetzungsprocess der oxydirten Eisenvitriollösung nach der Gmelin'schen Gleichung (siehe Tabelle X).

Tabelle X.

\begin{tabular}{|c|c|c|c|c|c|c|}
\hline & \multicolumn{3}{|c|}{ Niederschlag } & \multicolumn{3}{|c|}{ Lösung } \\
\hline & \multicolumn{2}{|c|}{ beobachtet } & \multirow[t]{2}{*}{ berechnet } & \multicolumn{2}{|c|}{ beobachtet } & \multirow[t]{2}{*}{ berechnet } \\
\hline & a. & b. & & a. & b. & \\
\hline $\mathrm{Fe}_{2} \mathrm{O}_{3}$ & $0,1199 \mathrm{~g}$ & $0,1156 \mathrm{~g}$ & $0,1151 \mathrm{~g}$ & $0,1712 \mathrm{~g}$ & $0,1730 \mathrm{~g}$ & $0,1727 \mathrm{~g}$ \\
\hline $\mathrm{SO}_{3}$ & 0,0289 & 0,0262 & 0,0288 & 0,2556 & 0,2578 & 0,2590 \\
\hline
\end{tabular}

Die Tabelle IX zeigt, dass immer der Schwefelsäuregehalt des Waschwassers relativ grösser ist als der des Filtrates. Es erklärt sich dies durch die Thatsache, dass die Niederschläge von basischem Ferrisulfat beim Waschen Schwefelsäure an das Waschwasser abgeben. Daher wurde in der vorstehenden, sowie in der nachfolgenden Tabelle der Schwefelsaureuberschuss im Waschwasser dem Schwefelsäuregehalte des Niederschlages zugerechnet.

a) Die Thatsache, dass eine oxydirle Eisenvitriollösung, welche $1 \mathrm{~g}$ Eisenvitriol in mehr als $50 \mathrm{ccm}$ Wasser gelöst enthielt, nach der Zersetzung einen grösseren Gehalt an Schwefelsäure aufweist, als dem normalen Ferrisulfat entspricht, dürfte nach dem im vorigen Paragraphen Gesagten darin ibre Erklärung finden, dass in diesen Fällen auch das normale Ferrisulfat schon theilweise nach der Gleichung: $3 \mathrm{Fe}_{2} \mathrm{~S}_{3} \mathrm{O}_{12}+8 \mathrm{aq}=\mathrm{Fe}_{6} \mathrm{SO}_{12}+$ $8 \mathrm{H}_{2} \mathrm{SO}_{4}$ zersetzt wurde. Die Lösung enthält somit $\mathrm{Fe}_{2} \mathrm{~S}_{3} \mathrm{O}_{12}$ und wechselnde Mengen freier Schwefelsäure und der Niederschlag ist ein Gemenge von $x \mathrm{Fe}_{4} \mathrm{SO}_{9}+y \mathrm{Fe}_{6} \mathrm{SO}_{12}$.

Die unter dieser Vorausselzung berecbnete Zusammensetzung von Lösung und Niederschlag stimmt mit den Beobachtungen, wie Tabelle XI lehrt, recht gut uberein. Bei dieser Berechnung wurden nur die Zahlen bertucksichtigt, welche bei achttăgiger Dauer der Reaction erhalten worden sind.

(Siehe nebenstehende Tabelle.)

Der Gang der Rechnung war folgender: Wenn die Zersetzung nach der Gmelin'schen Gleichung verläuft, sollen $0,1727 \mathrm{~g} \mathrm{Fe}_{2} \mathrm{O}_{3}$ in der Lösung sein. In der Wirklichkeit sind aber weniger in der Lösung. Die Differenz im Eisengebalte ist als $\mathrm{Fe}_{6} \mathrm{SO}_{12}$ dem Niederschlage zuzuzählen, der ursprünglich $0,1151 \mathrm{~g} \mathrm{Fe}_{2} \mathrm{O}_{3}$ und $0,0288 \mathrm{~g} \mathrm{SO}_{3}$ enthielt. Der Gehalt der Lösung an Schwefelsäure berechnet sich als Summe aus dem Schwefelsäuregehalte 
Tabelle XI.

\begin{tabular}{|c|c|c|c|c|c|c|c|c|}
\hline & & \multicolumn{3}{|c|}{ Beobachlet } & \multicolumn{3}{|c|}{ Berechnet } & \multirow{2}{*}{$\begin{array}{l}\text { Zersetzt } \\
\text { wurden }\end{array}$} \\
\hline & & $\mathrm{Fe}_{2} \mathrm{O}_{3}$ & $\mathrm{SO}_{3}$ & $\left|\mathrm{Fe}_{2} \mathrm{O}_{3}: \mathrm{SO}_{3}\right|$ & $\mathrm{Fe}_{2} \mathrm{O}_{3}$ & $\mathrm{SO}_{3}$ & $\mathrm{Fe}_{2} \mathrm{O}_{3}: \mathrm{SO}_{3} \mid$ & \\
\hline 4 & $\begin{array}{l}\text { Filtrat } \\
\text { Niederschlag }\end{array}$ & $\begin{array}{l}0,1644 \mathrm{~g} \\
0,1257\end{array}$ & $\begin{array}{l}0,2548 \mathrm{~g} \\
0,0307\end{array}$ & $\left|\begin{array}{ll}1 & : 3,10 \\
2,05 & : 1\end{array}\right|$ & $\left|\begin{array}{l}0,1644 \mathrm{~g} \\
0,1234\end{array}\right|$ & $\begin{array}{l}0,2578 \mathrm{~g} \\
0,0303\end{array}$ & $\left|\begin{array}{ll}1 & : 3,14 \\
2,04 & : 1\end{array}\right|$ & $4,81 \%$ \\
\hline $\mathbf{5}$ & $\begin{array}{l}\text { Filtrat } \\
\text { Niederschlag }\end{array}$ & $\begin{array}{l}0,1555 \\
0,1332\end{array}$ & $\begin{array}{l}0,2557 \\
0,0306\end{array}$ & $\left|\begin{array}{ll}1 & : 3,29 \\
2,1 & 6: 1\end{array}\right|$ & 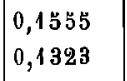 & $\begin{array}{l}0,2564 \\
0,0317\end{array}$ & $\left|\begin{array}{ll|}1 & : 3,30 \\
2,09 & : 1\end{array}\right|$ & 10 \\
\hline 6 & $\begin{array}{l}\text { Filtrat } \\
\text { Niederschlag }\end{array}$ & $\begin{array}{l}0,1471 \\
0,1439\end{array}$ & $\begin{array}{l}0,2534 \\
0,0329\end{array}$ & $\left|\begin{array}{ll}1 & : 3,44 \\
2,19 & : 1\end{array}\right|$ & $\begin{array}{l}0,1471 \\
0,1407\end{array}$ & $\begin{array}{l}0,2548 \\
0,0334\end{array}$ & $\left|\begin{array}{ll|}1 & : 3,47 \\
2,13 & : 1\end{array}\right|$ & 14,82 \\
\hline 7 & \begin{tabular}{|l} 
Filtrat \\
Niederschlag
\end{tabular} & $\begin{array}{l}0,1372 \\
0,1538\end{array}$ & $\begin{array}{l}0,2514 \\
0,0340\end{array}$ & $\left|\begin{array}{ll}1 & : 3,67 \\
2,26 & : 1\end{array}\right|$ & $\begin{array}{l}0,1372 \\
0,1506\end{array}$ & $\begin{array}{l}0,2530 \\
0,0347\end{array}$ & \begin{tabular}{ll|}
1 & $: 3,69$ \\
2,17 & $: 1$
\end{tabular} \mid & 20,56 \\
\hline 8 & $\begin{array}{l}\text { Filtrat } \\
\text { Niederschlag }\end{array}$ & $\begin{array}{l}0,1312 \\
0,1591\end{array}$ & $\begin{array}{l}0,2465 \\
0,0379\end{array}$ & $\begin{array}{ll}1 & : 3,76 \\
2,10 & : 1\end{array}$ & $\begin{array}{l}0,1312 \\
0,1566\end{array}$ & $\begin{array}{l}0,2520 \\
0,0357\end{array}$ & $\begin{array}{ll}1 & : 3,85 \\
2,22 & : 1\end{array} \mid$ & 24,41 \\
\hline
\end{tabular}

des noch gelösten normalen Ferrisulfates und der durch die partielle Zersetzung desselben nach obiger Gleichung freigewordenen Schwefelsäure.

Als Beispiel diene Versuch 4. In der Lösung sollen sein $0,1727 \mathrm{~g} \mathrm{Fe}_{2} \mathrm{O}_{3}$; gelöst sind $0,1644 \mathrm{~g} \mathrm{Fe}_{2} \mathrm{O}_{3}$. Differenz ist $0,0083 \mathrm{~g} \mathrm{Fe}_{2} \mathrm{O}_{3}$. Diese Eisenmenge bindet als $\mathrm{Fe}_{6} \mathrm{SO}_{12}$ $0,0014 \mathrm{~g} \mathrm{SO}_{3}$ und gab an die Lösung $0,0112 \mathrm{~g} \mathrm{SO}_{3}$ ab. Da die gelösten $0,1644 \mathrm{~g} \mathrm{Fe}_{2} \mathrm{O}_{3}$ als $\mathrm{Fe}_{2} \mathrm{~S}_{3} \mathrm{O}_{12} 0,2466 \mathrm{~g} \mathrm{SO}$ binden, so soll in der Lösung sein : $0,1644 \mathrm{~g} \mathrm{Fe}_{2} \mathrm{O}_{3}$ und 0,2466 $+0,0112 \mathrm{~g}=0,2578 \mathrm{~g} \mathrm{SO}_{3}$; der Niederschlag enthält $0,1151+0,0083=0,1234 \mathrm{~g} \mathrm{Fe}_{2} \mathrm{O}_{3}$ und $0,0288+0,0014=0,0302 \mathrm{~g} \mathrm{SO}_{3}$.

Wieviel normales Ferrisulfat jedesmal zerselzt wurde, giebt in vorstehender Tabelle die letzte Columne in Procenten ausgedrückt an.

Hervorzuheben ist, dass bei diesen Versuchen die Zersetzung des normalen Ferrisulfates schon bei viel grösseren Concentrationen eintrilt, als hei den früheren Verdunnungsversuchen. Dort wurde als Verdünnungsgrenze, hei welcher sich das normale Ferrisulfat zu zersetzen anfängl, der Gehalt von $0,1 \mathrm{~g} \mathrm{Fe}_{2} \mathrm{~S}_{3} \mathrm{O}_{12}$ in ca. $50 \mathrm{ccm}$ Wasser angegeben. Hier entspricht der

Versuch $\mathrm{Nr}$. 4 einer Verdunnung von $0,1 \mathrm{~g} \mathrm{Fe}_{2} \mathrm{~S}_{3} \mathrm{O}_{12}$ in $17,37 \mathrm{ccm}$ Wasser

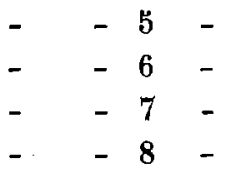

b. Die nächste Frage, welche zu beantworten ist, betriflt das Auftrelen der basischen Ferrisulfate in den concentrirteren Lösungen des oxydirten Eisenvitriols. Um diese Erscheinung zu erklären, kann man zwei Annahmen machen :

1) Ist es möglich, dass bei einer Concentration, die grösser ist als $1 \mathrm{~g}$ Eisenvitriol in $50 \mathrm{ccm}$ Wasser, nicht mehr die ganze Menge des anfänglich gebildeten Salzes $\mathrm{Fe}_{2} \mathrm{~S}_{2} \mathrm{O}_{9}$, sondern nur ein Theil desselben, dessen Grösse 
sich als Function der Concentration darstellt, in $\mathrm{Fe}_{2} \mathrm{~S}_{3} \mathrm{O}_{12}$ und $\mathrm{Fe}_{4} \mathrm{SO}_{9}$ zerselzt wird.

2) Wäre es auch denkbar, dass das durch die Zersetzung der Gosammtmenge von $\mathrm{Fe}_{2} \mathrm{~S}_{2} \mathrm{O}_{9}$ entstandene normale Ferrisulfat einen Theil des Niederschlages wieder auflöst und zwar um so mebr, je concentrirter die Lösung ist.

In beiden Fällen müssle der Niederschlag stets die Zusammensetzung $\mathrm{Fe}_{4} \mathrm{SO}_{9}$ haben. Fur die letztere Annahme könnte die Thatsache in's Feld gefuhrt werden, dass Lösungen von normalem Ferrisulfat, wie in einem späteren Paragraphen gezeigt werden wird, thatsächlich Eisenhydroxyd und basisches Ferrisulfat aufzulösen im Stande sind.

Gegen dieselbe und zugleich fur die erste Annahme spricht der Umstand, dass der Eisengehalt der Lösung bei längerem Stehen nicht, wie die vorige Annahme verlangen wurde, zu-, sondern abnimmt und dass sich gleichzeitig der Exponent des Molekularverhältnisses $\mathrm{SO}_{3}: \mathrm{Fe}_{2} \mathrm{O}_{3}$ in der Lösung erhöht. Die Zerselzung des basischen Ferrisulfates $\mathrm{Fe}_{2} \mathrm{~S}_{2} \mathrm{O}_{9}$ erfolgt in Lösungen nicht plötzlich, sondern allmählich und zwar so lange, bis ein dem Concentrationsgrade der Lösung entsprechender chemischer Gleichgewichtszustand hergestellt ist.

Wenn man von den beobachteten Exponenlen des Verhältnisses $\mathrm{SO}_{3}$ : $\mathrm{Fe}_{2} \mathrm{O}_{3}$ ausgeht, so wären in der Lösung enthalten:

Tabelle XII.

\begin{tabular}{r|l|l}
\hline & Nach zwölfstündigem Stehen & Nach achttägigem Stehen \\
\hline \hline bei Versuch 1 & $36 \mathrm{Fe}_{2} \mathrm{~S}_{3} \mathrm{O}_{12}+20 \mathrm{Fe}_{2} \mathrm{~S}_{2} \mathrm{O}_{9}$ & $54 \mathrm{Fe}_{2} \mathrm{~S}_{3} \mathrm{O}_{12}+20 \mathrm{Fe}_{2} \mathrm{~S}_{2} \mathrm{O}_{9}$ \\
$-\quad-\quad 2$ & $45-20-$ & $78-$
\end{tabular}

Es wurden demnach zersetzt:

Tabelle XIII.

\begin{tabular}{|c|c|c|c|}
\hline & Nach zwölfst ündigem Stehen & Nach achttägigem Stehen & Differenz \\
\hline bei Versuch 1 & $75,00 \% \mathrm{Fe}_{2} \mathrm{~S}_{2} \mathrm{O}_{9}$ & $81,81 \% \mathrm{Fe}_{2} \mathrm{~S}_{2} \mathrm{O}_{9}$ & $6,81 \%$ \\
\hline$-\quad-2$ & $78,94 \quad-$ & $86,66 \quad-$ & 7,72 \\
\hline
\end{tabular}

In der verdunnteren Lösung 2 ist also nicht nur innerbalb 24 Stunden mehr $\mathrm{Fe}_{2} \mathrm{~S}_{2} \mathrm{O}_{9}$ zersetzt worden als in der concentrirteren Lösung 1, sondern auch innerhalb 8 Tagen fiel noch ein grösserer Procentsatz der nachträglichen Zersetzung anheim.

Versucht man auf Grund obiger Annabmen die Constilution von Lösung und Niederschlag zu ermilteln, so ergiebt sich — vergl. Tabelle XIV — eine befriedigende Uehereinstimmung zwischen Beobachtung und Rechnung. 
Beitr. zur Kenntn. der chem. Constit. u. der Genese der nalürl. Eisensulfate. I. 227

Tabelle XIV.

\begin{tabular}{|c|c|c|c|c|c|c|c|c|}
\hline & \multicolumn{4}{|c|}{ Zwölfstündige Reactionsdauer } & \multicolumn{4}{|c|}{ Achtlägige Reaclionsdauer } \\
\hline & \multicolumn{2}{|c|}{ beobachtet } & \multicolumn{2}{|c|}{ berechnet } & \multicolumn{2}{|c|}{ beobachtet } & \multicolumn{2}{|c|}{ berechnet } \\
\hline & $\mathrm{Fe}_{2} \mathrm{O}_{3}$ & $\mathrm{SO}_{3}$ & $\mathrm{Fe}_{2} \mathrm{O}_{3}$ & $\mathrm{SO}_{3}$ & $\mathrm{Fe}_{2} \mathrm{O}_{3}$ & $\mathrm{SO}_{3}$ & $\mathrm{Fe}_{2} \mathrm{O}_{3}$ & $\mathrm{SO}_{3}$ \\
\hline fLösung & 0,1985 & 0,2623 & 0,2015 & 0,2662 & 0,1938 & 0,2644 & 0,1936 & 0,2642 \\
\hline TNiederschlag & 0,0881 & 0,0219 & 0,0863 & 0,0216 & 0,0923 & 0,0218 & 0,0942 & 0,0236 \\
\hline Joösung & 0,1950 & 0,2618 & 0,1969 & 0,2651 & 0,1866 & 0,2599 & 0,1880 & 0,2628 \\
\hline Niederschlag & 0,0976 & 0,0223 & 0,0909 & 0,0227 & 0,1052 & 0,0239 & 0,0998 & 0,0250 \\
\hline
\end{tabular}

\$. 5. Das Verhalten des Eisenvitriols in einer Sauerstoffatmosphäre. Der geringe Gehalt der mir zur Verfügung stebenden Wasserstoffsuperoxydlösung an $\mathrm{H}_{2} \mathrm{O}_{2}$ gestattete mir leider nicht, die obigen Experimente mit stärker concentrirlen Eisenvitriollösungen fortzuselzen. Ich versuchte daher eine weitere Bestätigung der am Schlusse des vorigen Paragraphen gemachten Annahmen dadurch zu erlangen, dass ich Eisenvitriol der Einwirkung einer Sauerstoffatmosphäre aussetzte.

$\mathrm{Zu}$ diesem $Z$ wecke wurde Eisenvitriol in einer Glasschale unter einen mit Sauerstoff gefullten Recipienten gebracht. Dies geschah auf folgende Weise. Ein grosser tubulirter Recipient, dessen Tubulatur durch einen eingeschliffenen Stöpsel verschliessbar war, wurde nach Entfernung des Stöpsels uber eine auf dem Wasser schwimmende, Eisenvitriol enthaltende Glasschale gestülpt und dann so tief in das Wasser eingetaucht, dass möglichst viel Luft daraus verdrängt wurde. Hierauf wurde die Tubulatur mittelst eines gut eingefetteten Stöpsels verschlossen, der Recipient auf die Brucke der pneumatischen Wanne gehoben und mit Sauerstoff gefüllt.

Bei den zahlreichen auf diese Weise ausgefuhrten Versuchen machte ich die Wahrnehmung, dass die Oxydation des Eisenvitriols nur im unverstäubten Zustande erfolgt. Denn als ich verstäubten Eisenvitriol unter denselben Bedingungen der Einwirkung einer Sauerstoffatmosphäre aussetzte, beobachtete ich eine beginuende Oxydation erst dann, als sich der verstäubte Eisenvitriol durch Wasseraufnahme zu normalem Eisenvitriol mit 7 Molekulen Wasser regenerirt hatte. Diesen Vorgang verrielh der allmähliche Wechsel der Farbe des Pulvers vom ursprunglichen Weiss in's Grün. Wurde dagegen durch Einschalten einer $2 \mathrm{~cm}$ dicken Oelschicht zwischen Sauerstoff und Wasser dem verstäublen Vitriol die Möglichkeit der Wasseraufnahme genommen, so trat in derselben Zeit, in welcher der Eisenvitriol des vorigen Versuches schon ganz braun geworden war, hier noch keine merkliche Spur einer Oxydation auf.

Ferner beobachtete ich, dass wahrend der Oxydation des Eisenvitriols stets eine theilweise Verflussigung des Salzes in Folge von Wasseraufnahme stattfand. Es waren nun zwei Möglichkeiten offen, um diese Erscheinung zu 
erklären. Einerseils konnle die Wasseraufnahme eine rein hygroskopische Wirkung des Pulvers sein, andererseits konnte sie auch eine unmittelbare Folge des Oxydationsprocesses sein. Wurde nämlich beispielsweise die Oxydation des Eisenvitriols nach der Gleichung:

$$
4\left[\mathrm{FeSO}_{4}+7 \mathrm{aq}\right]+2 \mathrm{O}=\mathrm{Fe}_{4} \mathrm{~S}_{3} \mathrm{O}_{15}+13 \mathrm{aq}+\mathrm{H}_{2} \mathrm{SO}_{4}+14 \mathrm{IH}_{2} \mathrm{O}
$$

erfolgen, so wäre das Auftreten von Flüssigkeit zur Genuge erklärt.

Um nun all' diese Fragen zu entscheiden, wurden grosse Stücke Eisenvitriol mittelst eines Glasdreieckes und eines Filterpapierblattes in die Glasschale gelegt. Auf diese Weise war unter dem Filterpapiere ein leerer Raum geschaffen worden, in den die sich bildende Flüssigkeit fltriren konnte. Der Eisenvitriol wurde dadurch möglichst schuell der Einwirkung der sich bildenden Flüssigkeit entzogen. Nach fünf Monaten halte sich unter dem Filterpapiere eine kleine Menge einer rothbraunen Flüssigkeit angesammelt, die im ccm enthielt :

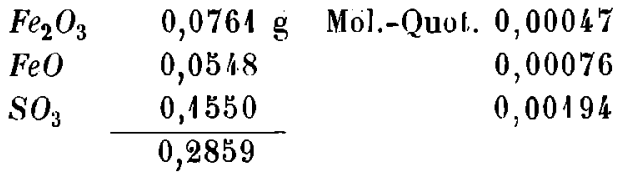

Es war demnach nicht wässerige Schwefelsäure durchfiltrirt, sondern es waren Eisenvitriol und ein Ferrisulfat gelöst worden. Das Ferrisulfat hatte die Zusammensetzung $\mathrm{Fe}_{4} \mathrm{~S}_{5} \mathrm{O}_{21}$, denn nach Abzug der an das Eisenoxydul gebundenen Schwefelsäure stellte sich das Molekularverhältniss des Schwefelsäurerestes zum Eisenoxyd wie 2,51: 1 .

Dieser Versuch lehrt also, dass selbst dann, wenn bei der Oxydation des Eisenvitriols nur Wasserdunst zugegen ist, sich nicht bleibend $\mathrm{Fe}_{2} \mathrm{~S}_{2} \mathrm{O}_{9}$ bildet, sondern dass sofort eine Zerselzung dieses Sulfates eintritl. Ferner geht daraus hervor, dass die beobachtete Wasseranziehung nicht davon berrührt, dass sich freie Schwefelsäure abspaltet, sondern dass diese bygroskopische Wirkung den neugebildeten Ferrisulfaten zugeschrieben werden muss.

Die Zersetzung verläuft hier ganz so, wie in den fruberen Versuchen. Es bildet sich $\mathrm{Fe}_{2} \mathrm{~S}_{2} \mathrm{O}_{9}$, das jedoch wegen der grossen Concentration der Lösung nur mehr theilweise in $\mathrm{Fe}_{2} \mathrm{~S}_{3} \mathrm{O}_{12}$ und $\mathrm{Fe}_{4} \mathrm{SO}_{9}$ zerfällt wird.

Den exacten Beweis hierfür erbringt nachstehender Versuch. $3,0998 \mathrm{~g}$ Eisenvitriol wurden vier Monate lang in einer Glasschale der Einwirkung einer Sauerstoffatmosphäre ausgesetzt. Nach Ablauf dieser Frist wurde die Schale aus dem Recipienten herausgenommen und die rothbraune Lösung durch Filtration vom ockergelben Niederschlage getrennt. Diese unverdunnte Lösung ist in der nachstehenden Tabelle XV unter „Filtrat " verstanden. Hierauf wurde der Niederschlag mit kaltem Wasser gewaschen, bis das Waschwasser farblos durchlief (1. Waschwasser), dann wurde aber 
das Waschen noch so lange fortgesetal, bis im Waschwasscr kein Eisen mehr nachweisbar war (2. Waschwasser). Der unlösliche Rückstand wurde lufttrocken vom Filter herabgenommen und besonders analysirt. Die am Filter haften gebliebenen Reste wurden in Salzsäur'e gelöst und die darin gefundenen Eisen- und Schwefelsäuremengen wurden unter wFillerrückstand " in die Tabelle eingestellt.

Tabelle XV.

\begin{tabular}{l|c|c|c}
\hline & $F e O$ & $\mathrm{Fe}_{2} \mathrm{O}_{3}$ & $\mathrm{SO}$ \\
\hline \hline Filtrat & $0, \dot{0} 467 \mathrm{~g}$ & $0,2612 \mathrm{~g}$ & $0,3731 \mathrm{~g}$ \\
1. Waschwasser & 0,0627 & 0,3162 & 0,4584 \\
2. $\quad-$ & - & 0,0128 & 0,0183 \\
Niederschlag & - & 0,1516 & 0,0354 \\
Filterrückstand & - & 0,0289 & 0,0068 \\
\hline & $0,1094 \mathrm{~g}$ & $0,7707 \mathrm{~g}$ & $0,8920 \mathrm{~g}$
\end{tabular}

Die Verdunnung war 7,34 ccm, d. h. in dieser Wassermenge war $1 \mathrm{~g}$ Substanz gelöst. Innerhalb vier Monaten war somit noch nicht aller Eisenvitriol oxydirt worden. Die Menge des unoxydirten Vitriols beträgt 0,4228 $\mathrm{g}$ $=13,64 \%$. Bringt man den noch unveränderten Eisenvitriol in Abzug, so lautet dann obige Tabelle :

T.abelle. XVI.

\begin{tabular}{l|l|l|c}
\hline & $\mathrm{Fe}_{2} \mathrm{O}_{3}$ & $\mathrm{SO}_{3}$ & $S \mathrm{O}_{3} / \mathrm{Fe}_{2} \mathrm{O}_{3}$ \\
\hline \hline Filtrat & $0,2612 \mathrm{~g}$ & $\mathbf{0 , 3 2 1 3 \mathrm { g }}$ & 2,46 \\
1. Waschwasser & 0,3162 & 0,3887 & 2,46 \\
2. & 0,0128 & 0,0183 & 2,86 \\
Niederschlag in toto & 0,1805 & 0,0422 & $2,14 / 1$ \\
\hline \multicolumn{1}{c|}{ Summe } & $0,7707 \mathrm{~g}$ & $0,7705 \mathrm{~g}$ &
\end{tabular}

Wenn die Annahme, dass in concentrirten Lösungen nur mehr ein Theil des ursprünglichen Ferrisulfates $\mathrm{Fe}_{2} \mathrm{~S}_{2} \mathrm{O}_{3}$ zersetzt wird, durch diesen Versuch eine Bestätigung finden soll, dann müssen jedem Molekül $\mathrm{Fe}_{4} \mathrm{SO}_{9}$ im Niederschlage 3 Moleküle $\mathrm{Fe}_{2} \mathrm{~S}_{3} \mathrm{O}_{12}$ in der Lösung entsprechen.

Berechnet man auf diese Weise die Zusammensetzung der Lösung und des Niederschlages, indem man vom beobachteten Eisengehalte des Niederschlages ausgeht, so gelangt man zu folgenden Zahlen : 
Tabelle XVII.

\begin{tabular}{|c|c|c|c|}
\hline & & $\mathrm{F}_{\mathrm{C}_{2}} \mathrm{O}_{3}$ & $\mathrm{SO}_{3}$ \\
\hline \multirow{3}{*}{ Lösung } & $\begin{array}{l}\mathrm{Fe}_{2} \mathrm{~S}_{2} \mathrm{O}_{9} \\
\mathrm{Fe}_{2} \mathrm{~S}_{3} \mathrm{O}_{12}\end{array}$ & $\begin{array}{l}0,3195 \mathrm{~g} \\
0,2707\end{array}$ & $\begin{array}{l}0,3195 \mathrm{~g} \\
0,4061\end{array}$ \\
\hline & $\begin{array}{l}\text { Summe berechnet } \\
-\quad \text { beohachtet }\end{array}$ & $\begin{array}{l}0,5901 \mathrm{~g} \\
0,5902\end{array}$ & $\begin{array}{l}0,7256 \mathrm{~g} \\
0,7267\end{array}$ \\
\hline & Beob. - Rechn. & $+0,0001 \mathrm{~g}$ & $+0,0011 \mathrm{~g}$ \\
\hline \multirow{2}{*}{$\begin{array}{l}\text { Nieder- } \\
\text { schlag }\end{array}$} & $\begin{array}{c}\mathrm{Fe}_{4} \mathrm{SO}_{9} \text { berechnet } \\
-\quad \text { beobachtet }\end{array}$ & $\begin{array}{l}0,1805 \mathrm{~g} \\
0,1805\end{array}$ & $\begin{array}{l}0,0451 g \\
0,0438\end{array}$ \\
\hline & Beob. -- Rechn. & $0,0000 \mathrm{~g}$ & $-0,0043 \mathrm{~g}$ \\
\hline
\end{tabular}

Die Uebereinstimmung zwischen Beobachtung und Rechnung ist fast eine absolute. Der Gehalt der Lösung an Schwefelsäure erscheinl hier um $0,0016 \mathrm{~g}$ kleiner als in Tabelle XVI, weil der Ueberschuss an Schwefelsüure im Waschwasser 2 dem Niederschlage zugerechnet wurde.

Die wichtigsten Ergebnisse vorstehender Untersuchungen sind in Kur'ze folgende:

1. Der Eisenvitriol verstăubt, wenn er lange genug an der Luft gelegen ist, $\mathbf{z u} \mathrm{FeSO}_{4}+$ aq.

2. Die nebenher schreitende Oxydation ist von ganz untergeordneter Bedeutung.

3. Die Zersetzung einer oxydirten Eisenvitriollösung ist ebenso wie die Zersetzung einer verdünnten Lösung von $\mathrm{Fe}_{2} \mathrm{~S}_{3} \mathrm{O}_{12}$ kein plötzlicher, sondern ein allmählicher Vorgang. Die Zeit, welche vom Beginne der Zersetzung bis zur Herstellung des chemischen Gleichgewichtes verstreicht, ist im Allgemeinen bei oxydirten Eisenvitriollösungen um so länger, bei verdúnnten Lösungen von Ferrisulfat jedoch um so kürzer, je verdünnter die Lösung ist.

4. Unter Berúcksichtigung dieses Umstandes sind die Producte der Zersetzung lediglich Functionen der Concentration der oxydirten Eisenvitriollösung.

5. Nur wenn $1 \mathrm{~g}$ Eisenvitriol in circa $50 \mathrm{ccm}$ Wasser gelöst ist, enthält die Lösung dauernd normales Ferrisulfat.

6. Bei grösserer Concentration sind basische Salze gelöst, bei geringerer Concentration ist neben normalem Ferrisulfat noch freie Schwefelsäure in der Lösung enthalten.

7. Das Auftreten basischer Sulfate in der Lösung erklärt sich dadurch, dass in concentrirteren Lösungen immer nur ein zur Concentration umgekehrt proportionaler Theil des ursprunglichen Salzes $\mathrm{Fe}_{2} \mathrm{~S}_{2} \mathrm{O}_{\mathrm{g}}$ der Zersetzung anheimfällt, 
8. Die Zersetzung erfolgh nach der Gleichung $5 \mathrm{Fe}_{2} \mathrm{~S}_{2} \mathrm{O}_{9}=3 \mathrm{Fe}_{2} \mathrm{~S}_{3} \mathrm{O}_{9} \dashv-$ $\mathrm{Fe}_{4} \mathrm{SO}_{9}$.

9. Das Auftreten freier Schwefelsäure neben normalem Ferrisulfat in verdünteren Lösungen ist eine Folge der Thatsache, dass sich Lösungen von normalem Ferrisulfat, wenn sie über eine gewisse Grenze verdunnt werden, auch in der Kälte parliell in $\mathrm{Fe}_{6} \mathrm{SO}_{12}$ und freie Schwefelsäure spalten.

10. Auch in diesem Falle wird um so mehr Salz zersetzt, je verdunnter die Lösung isl.

11. Die bei der Zersetzung einer oxydirten Eisenvitriollösung sich bildenden Niederschläge bestehen nur dann, wenn in der Lösung normales Ferrisulfat oder basisches Ferrisulfat enthalten ist, aus $\mathrm{Fe}_{4} \mathrm{SO}_{9}$. Bei stärkeren Verdünnungen sind sie Gemenge von $\mathrm{Fe}_{4} \mathrm{SO}_{9}$ und $\mathrm{Fe}_{6} \mathrm{SO}_{12}$ in wechselnden Mengen.

12. Es ist bisher noch nicht gelungen, durch Oxydation von Eisenvilriollösungen die Verbindung $\mathrm{Fe}_{2} \mathrm{~S}_{2} \mathrm{O}_{9}$ allein und dauernd in der Lösung zu erbalten. Das basischeste Salz, welches bis jetzt in Lösungen beobachtet wurde, nähert sich in seiner Zusammensetzung der Formel $F_{10} S_{12} O_{51}=$ $3 \mathrm{Fe}_{2} \mathrm{~S}_{2} \mathrm{O}_{9}+2 \mathrm{Fe}_{2} \mathrm{~S}_{3} \mathrm{O}_{12}$.

Gzernowitz, den 8. Mai 1898. 